\title{
AURKB, CHEKI and NEK2 as the Potential Target Proteins of Scutellaria barbata on Hepatocellular Carcinoma: An Integrated Bioinformatics Analysis
}

\author{
Chaoyuan Huang (iD) \\ Hu Luo' \\ Yuancheng Huang (D) \\ Chongkai Fang' \\ Lina Zhao ${ }^{2}$ \\ Peiwu $\mathrm{Li}^{2}$ \\ Chong Zhong (iD ${ }^{3}$ \\ Fengbin Liu (iD ${ }^{2,4}$ \\ 'The First Clinical Medical School, \\ Guangzhou University of Chinese \\ Medicine, Guangzhou, People's Republic \\ of China; ${ }^{2}$ Department of gastroenterol- \\ ogy, The First Affiliated Hospital of \\ Guangzhou University of Chinese \\ Medicine, Guangzhou, People's Republic \\ of China; ${ }^{3}$ Department of Hepatobiliary \\ Surgery, The First Affiliated Hospital of \\ Guangzhou University of Chinese \\ Medicine, Guangzhou, People's Republic \\ of China; ${ }^{4}$ Department of gastroenterol- \\ ogy, Baiyun Hospital of the First Affiliated \\ Hospital of Guangzhou University of \\ Chinese Medicine, Guangzhou, People's \\ Republic of China
}

Objective: We aim to explore the potential anti-HCC mechanism of Scutellaria barbata through integrated bioinformatics analysis.

Methods: We searched active ingredients and related targets of Scutellaria barbata via TCMSP database, PubChem and SwissTargetPrediction database. Then, we identified HCC disease targets from GEO dataset by WGCNA. Next, the intersected targets of disease targets and drug targets were input into STRING database to construct PPI networking in order to obtain potential therapeutic targets of Scutellaria barbata. Cytoscape software was used to carry out network topology analysis of potential targets. We used the R package for GO analysis and KEGG analysis. Finally, we used AutoDock vina and PyMOL software for molecular docking.

Results: Sixteen active components from Scutellaria barbata were lastly selected for further investigation. A total of 442 component targets were identified from 16 active ingredients of Scutellaria barbata after the removal of duplicate targets. GSE45436 was selected for construction of WGCNA and screening of differentially expressed genes. A total of 354 genes were up-regulated in HCC samples and 100 were down-regulated in HCC patients. Twenty-one common genes were obtained by intersection and 10 critical targets were filtered for further investigation. The enrichment analysis showed that cell cycle, DNA replication, p53 signaling pathway were mainly involved. The molecular docking results showed that 4 potential combinations were with the best binding energy and molecular interactions.

Conclusion: AURKB, CHEK1 and NEK2 could be the potential target proteins of Scutellaria barbata in treating HCC. Cell cycle, DNA replication, p53 signaling pathway consist of the fundamental regulation cores in this mechanism.

Keywords: Scutellaria barbata, hepatocellular carcinoma, effector mechanism, network pharmacology, bioinformatics analysis, molecular docking

\section{Introduction}

Liver cancer is one of the malignant tumors with the highest incidence rates in the world, of which the incidence rate increases by approximately 700,000 patients worldwide each year, and over half of the global incidence and mortality of liver cancer occurs in China. ${ }^{1,2}$ Hepatocellular carcinoma (HCC) is the most common primary liver cancer subtype that contributes majority incidence of liver cancers worldwide. ${ }^{3}$ Epidemiologically, the number of NASH-related HCC cases
Correspondence: Fengbin Liu; Chong

Zhong

Email liufb163@vip.163.com;

zhongchong1732@gzucm.edu.cn 
is increasing, while that of HBV- or HCV-related cases is declining and that of alcohol-related cases remaining unchanged. ${ }^{4}$ Nevertheless, the burden of HCC would probably increase worldwide in the further as the population growth and aging expected in the next few decades. ${ }^{4}$ Hence, it is of great significance to explore new therapeutic targets for the treatment of HCC. Besides surgery, chemotherapy, interventional radiology, immunotherapy, molecular-targeted therapy and liver transplant, herbal products in Traditional Chinese Medicine (TCM) now have been considered as an upcoming therapeutic approach for prevention and treatment of HCC. ${ }^{5}$

Scutellaria barbata (Ban Zhi Lian in Chinese) is used as an immunomodulatory and antitumor agent in TCM. ${ }^{6}$ Scutellaria barbata is the whole dried grass of a labiate plant Scutellaria barbata D. Don, and has an extensive application for efficacy enhancement in cancer treatment in China and Asian countries. ${ }^{7}$ Effective ingredients of Scutellaria barbata have shown growth-inhibitory effects in various types of cancer, including liver cancer. ${ }^{8-10}$

Network pharmacology, first proposed in 2007, has become an effective assistant to systematically provide a novel strategy to research the mechanism of TCM formula with multiple compounds. ${ }^{11}$ Weighted gene coexpression network analysis (WGCNA) is a new tool that is adopted to identify co-expressed modules and hub genes. ${ }^{12}$ WGCNA can extract gene co-expression modules and contact it to the clinical feature. ${ }^{13}$ At present, WGCNA can be useful to identify candidate biomarkers or therapeutic targets of various diseases, and compare differentially expressed genes (DEGs) in order to help investigating the interactions among genes in different modules. ${ }^{14}$ Molecular docking technique is adopted to realize the drug-receptor interaction and orientation and approach of drug molecules to the target site. $^{15}$

In this study, we aim to explore the potential anti-HCC mechanism of Scutellaria barbata through network pharmacology and bioinformatics analysis. Furthermore, molecular docking was also performed to validate the binding interaction of the components in the binding pocket of the target proteins.

\section{Materials and Methods}

The flow chart of this study is shown in Figure 1.

\section{Screening and Preprocessing of Active Components from Scutellaria barbata}

By searching the Traditional Chinese Medicine Systems Pharmacology Database and Analysis Platform (TCMSP, https://tcmspw.com/tcmsp.php) database, the qualified components were collected. "Scutellaria barbata" was searched as Herb name in TCMSP, oral bioavailability (OB) $\geq 30 \%$ and drug-like (DL) $\geq 0.25$ were set in the Ingredients module, the selected molecule names were researched in PubChem database (https://pubchem.ncbi.nlm. nih.gov/) and the chemical components without PubChem CID were removed. The canonical SMILES strings and 3D structures of the related components were obtained from the PubChem database by PubchemCID and all the chemical structures were inputted into Excel as backup.

\section{Screening of Molecular Composition Target}

The canonical SMILES strings obtained from the PubChem database were uploaded to the SwissTargetPrediction server (http://www.swisstargetprediction.ch/) for molecular composition target prediction. The platform species is set to "Homo sapiens", and the predicted results of the platform were collected and classified. All predicted results are stored as CSV format. Furthermore, all of the results were dereprocessed, transferred to Excel format, and a molecular library was established.

\section{Sources of Clinical Data and Screening of Differentially Expressed Genes}

In order to further explore the clinical significance of the predicting target of Scutellaria barbata, the key word "liver cancer" was searched in Gene Expression Omnibus (GEO) datasets (https://www.ncbi.nlm.nih.gov/ gds), and the datasets with more than 100 samples were selected. In this study, the whole genome genes were detected, and the DEGs in the dataset were analyzed and screened. $|\log F C| \geq 1$ was used as the filtering threshold criteria of $\operatorname{logFC}$ and adj-P-value $<0.05$ as the corrected threshold of $P$-values. $\operatorname{LogFC}>1$ was regarded as upregulated genes in tumor samples while $\operatorname{LogFC}<-1$ would be considered as down-regulated genes in tumor samples. The heatmap and volcano plot of the screened genes were drawn by R software (Version 3.6.3). 


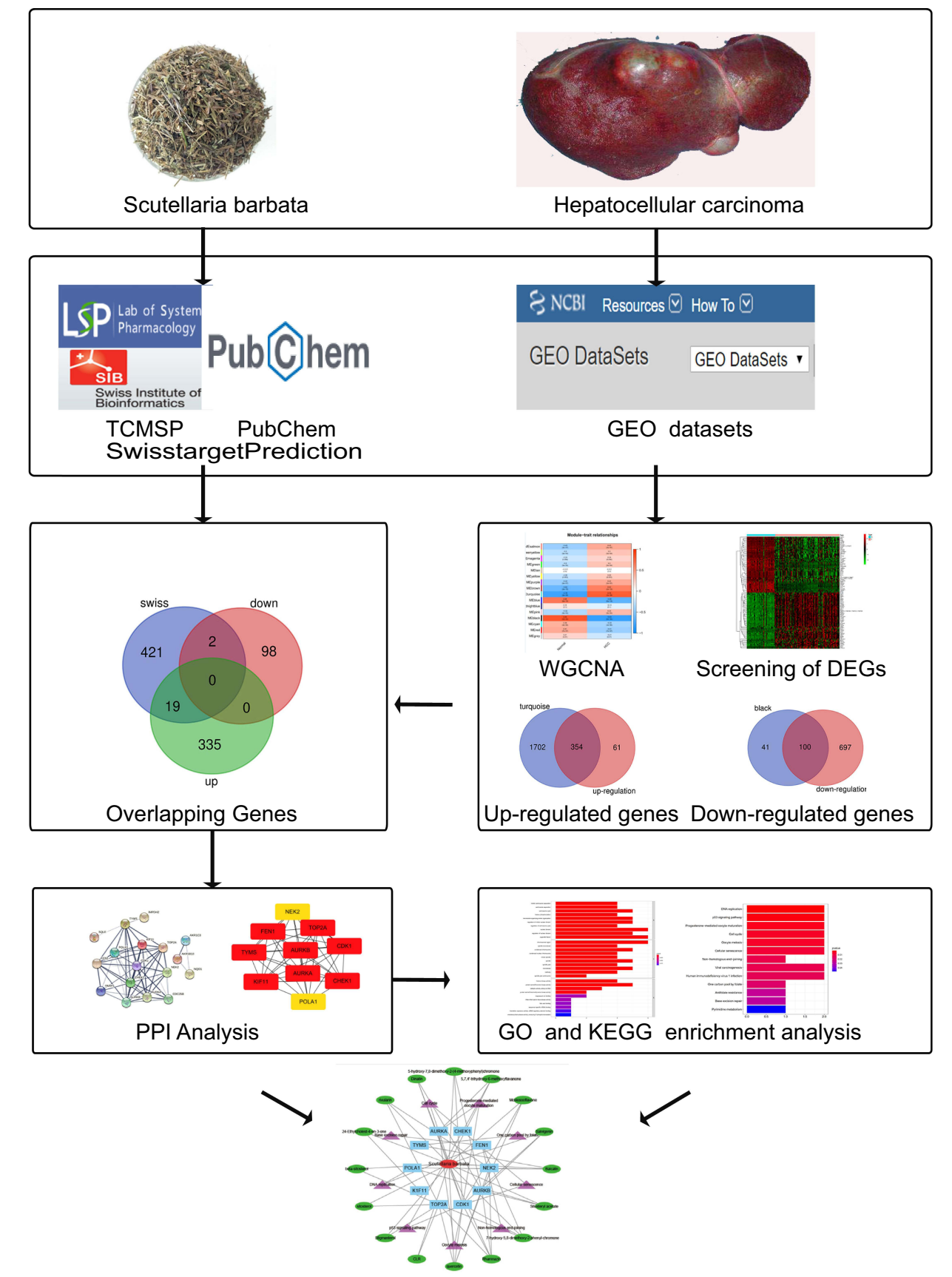

Construction of active component-Antihepatoma Target-PathwayNetwork

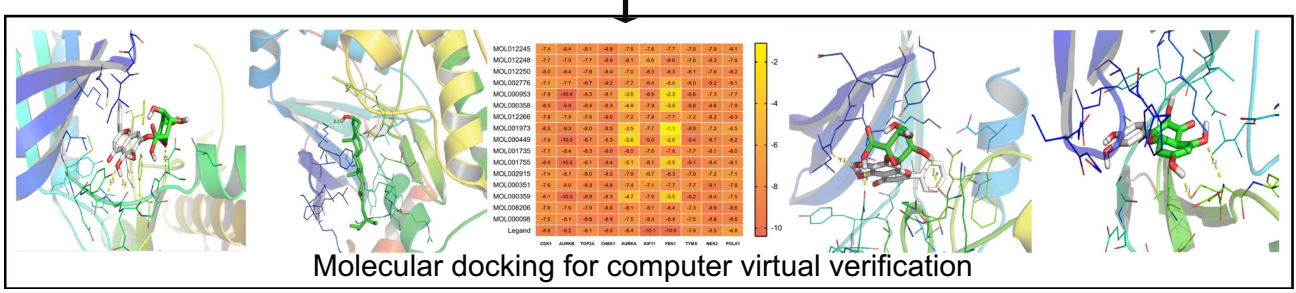

Figure I Workflow for exploring the molecular mechanisms of Scutellaria barbata against Hepatocellular carcinoma. 


\section{Construction of Gene Co-Expression Networks}

The R package "WGCNA" was used for finding clusters (modules) of highly correlated genes, for summarizing such clusters using the module eigengene or an intramodular hub gene. ${ }^{16}$ In this study, WGCNA construction and module detection were performed using the "WGCNA" $\mathrm{R}$ package and top $25 \%$ genes with the largest variance differences were used to construct weight gene coexpression networks in GSE45436. First, the gene expression data were used to obtain a Pearson correlation matrix between the genes. A soft threshold $\beta$ can improve strong correlations and weaken weak correlations. The power of $\beta=6$ (scale-free $\mathrm{R}^{2}=0.85$ ) was selected as the softthresholding parameter to ensure a scale-free network. Next, the linkage hierarchical clustering was produced by the topological overlap measure to carry out module detection and acquire the cluster dendrogram. Under the condition of setting minimum cluster size as 30, 16 modules were generated via Dynamic Tree Cut algorithm.

\section{Intersection of WGCNA Co-Expression Genes and DEGs}

In this study, the positive correlation modules that generated by WGCNA could be considered as up-regulated genes in tumor samples, while the negative correlation modules were regarded as down-regulated genes in tumor samples. The hub module with the highest positive correlation in normal and tumor patients matched with the differentially up-regulated genes, and the hub module with the highest negative correlation was intersected with the differentially down-regulated genes, the result was mapped into Venn diagram, and the overlapping genes obtained were used as HCC-related genes in this study.

\section{Searching for Overlapping Targets and Key Targets of Scutellaria barbata and $\mathrm{HCC}$}

The overlapping targets of Scutellaria barbata and HCC were found, and a Venn diagram was drawn. Next, the overlapping targets were inputted into String (http://stringdb.org/, version 11) for protein-protein interaction (PPI) analysis. The String database is a biological database for prediction of PPIs, with data from several sources. ${ }^{17}$ For further exploration, the minimum required confidence score was medium confidence (0.400), and disconnected nodes in the network would be hidden. The results were exported in tab-separated value (TSV) format once the protein interaction relationship was obtained. The PPI network was constructed via utilizing the network visualization software Cytoscape 3.8.1 (http://www.cytoscape.org/). CytoHubba is a tool used to identify hub objects and subnetworks from a complex interactome. ${ }^{18}$ CytoHubba provides 11 topological analysis methods including degree centrality (DC), Edge Percolated Component (EPC), Maximum Neighborhood Component (MNC), Density of Maximum Neighborhood Component (DMNC), Maximal Clique Centrality (MCC) and centralities based on shortest paths, such as Bottleneck (BN), closeness centrality (CC), betweenness centrality (BC), EcCentricity, Radiality, and Stress. Following the construction of a PPI network, hub genes were identified with the MCC algorithm. MCC algorithm can accurately identify hub genes in $\mathrm{HCC}^{19}$

\section{GO and KEGG Enrichment Analysis Potential Targets of Scutellaria barbata}

Gene Ontology (GO) and Kyoto Encyclopedia of Genes and Genomes (KEGG) enrichment analysis was performed to examine the enrichment of terms. The $\mathrm{R}$ package "ggplot2", “org.Hs.eg.db", "clusterProfiler" and "enrichplot" was used for GO and KEGG enrichment analysis. Enriched GO terms and KEGG pathways were identified with the cut-off criterion of $\mathrm{P}<0.05$.

\section{Construction of Active}

\section{Component-Antihepatoma Target-Pathway Network of Scutellaria barbata}

The network of active component of Scutellaria barbata-anti-hepatoma target - pathway was constructed by Cytoscape software. The node in the network represents Scutellaria barbata, active components of Scutellaria barbata, key target genes, gene enrichment pathway. The edge in the network is used to connect Scutellaria barbata and active components, active components and key target genes, key target genes and enrichment pathway. The whole network shows the relationship between Scutellaria barbata, active components, anti-hepatoma target and pathway, and explores the anticancer mechanism of Scutellaria barbata by constructing this network. 


\section{Molecular Docking}

The core target protein structures were downloaded from Protein Data Bank (PDB) database (http://www.rcsb.org). The water molecules were removed and the original ligands were separated via PyMol 1.8 software. After preservation, they were transferred into Autodock Tools 1.5.6 software and converted to "pdbqt" format. The mol2 structure of qualified components downloaded from TCMSP database, and transferred to Autodock Tools 1.5.6 software in order to convert to "pdbqt" format. The abovementioned two "pdbqt" format files were input into Autodock-vina 1.1.2 software for molecular docking. PyMol software was used to visualize the docking results and establish docking interaction pattern.

\section{Prognostic Value of the Potential Target Genes of Scutellaria barbata}

GEPIA2 (Gene Expression Profiling Interactive Analysis, http://gepia2.cancer-pku.cn/) was used to explore the prognostic values of the potential target genes in patients with HCC. We used the "Survival Analysis" module of GEPIA2 to obtain the OS (Overall Survival) and DFS (Disease FreeSurvival) Kaplan-Meier curve of potential target genes in HCC. The patients were grouped into high-expression and lowexpression according to the median expression of the potential target genes. Cutoff-high (50\%) and cutoff-low (50\%) values were used as the expression thresholds for splitting the highexpression and low-expression cohorts.

\section{Result}

\section{Identification of Targets of Scutellaria barbata}

A total of 94 components from Scutellaria barbata were found from the TCMSP database. 22 active components were available under the condition of $\mathrm{OB} \geq 30 \%$ and $\mathrm{DL} \geq 0.25$. Active components without PubChem CID were excluded due to the absence of canonical SMILES strings and 3D structures, which were significant for predicting the component targets. Sixteen active components were lastly selected for further investigation (Table 1). They were 7-hydroxy-5,8-dimethoxy-2-phenyl-chro-mone (MOL012250), 5-hydroxy-7,8-dimethoxy-2-(4-methoxyphenyl)chromone (MOL012248), 5,7,4'-trihydroxy -6-methoxyflavanone (5,7,4'-trihydroxy-6-methoxyflavanone,MOL012245), Baicalin (MOL002776), CLR (MOL000953), beta-sitosterol (MOL000358), rivularin (MOL012266), Sitosterylacetate (MOL001973), Stigmasterol (MOL000449), Dinatin (MOL001735), 24Ethylcholest-4-en-3-one (MOL001755), Salvigenin (MOL002915), Rhamnazin (MOL000351), sitosterol (MOL000359), Moslosooflavone (MOL008206), quercetin (MOL000098). Four hundred and forty-two component targets were identified from 16 active ingredients of Scutellaria barbata via SwissTargetPrediction database after the removal of duplicated targets. According to the prediction result of SwissTargetPrediction, quercetin may be highly combined with 100 targets such as NOX4, AVPR2, AKR1B1, XDH, MAOA, IGF1R, FLT3,

Table I Detail Information of 16 Compounds of Scutellaria barbata

\begin{tabular}{|c|c|c|c|c|c|c|}
\hline No. & Components & PubChemCID & CAS & Molecular ID & OB $\%$ & DL \\
\hline I & 5,7,4'-trihydroxy-6-methoxyflavanone & 5322074 & $94942-49-1$ & MOLOI 2245 & 36.63 & 0.27 \\
\hline 2 & 5-hydroxy-7,8-dimethoxy-2-(4-methoxyphenyl)chromone & 14353376 & $57096-03-4$ & MOLOI 2248 & 65.82 & 0.33 \\
\hline 3 & 7-hydroxy-5,8-dimethoxy-2-phenyl-chromone & 14825644 & N/A & MOLOI 2250 & 43.72 & 0.25 \\
\hline 4 & Baicalin & 64982 & $2 \mid 967-41-9$ & MOL002776 & 40.12 & 0.75 \\
\hline 5 & CLR & 5997 & $57-88-5$ & MOL000953 & 37.87 & 0.68 \\
\hline 6 & Beta-sitosterol & 222284 & $83-46-5$ & MOL000358 & 36.91 & 0.75 \\
\hline 7 & Rivularin & 13889022 & $70028-59-0$ & MOLOI 2266 & 37.94 & 0.37 \\
\hline 8 & Sitosteryl acetate & 5354503 & $915-05-9$ & MOL00I973 & 40.39 & 0.85 \\
\hline 9 & Stigmasterol & 5280794 & $83-48-7$ & MOL000449 & 43.83 & 0.76 \\
\hline 10 & Dinatin & 5281628 & |447-88-7 & MOL00I735 & 30.97 & 0.27 \\
\hline II & 24-Ethylcholest-4-en-3-one & 5484202 & $67392-96-5$ & MOL00I755 & 36.08 & 0.76 \\
\hline 12 & Salvigenin & $16127 \mid$ & $19103-54-9$ & MOL0029I5 & 49.07 & 0.33 \\
\hline 13 & Rhamnazin & 5320945 & $552-54-5$ & MOL00035I & 47.14 & 0.34 \\
\hline 14 & Sitosterol & 12303645 & $83-46-5$ & MOL000359 & 36.91 & 0.75 \\
\hline 15 & Moslosooflavone & 188316 & $3570-62-5$ & MOL008206 & 44.09 & 0.25 \\
\hline 16 & Quercetin & 5280343 & $117-39-5$ & MOL000098 & 46.43 & 0.28 \\
\hline
\end{tabular}


CYP19A1, EGFR, etc. Rhamnazin may have a high degree of binding with 74 targets such as $\mathrm{XDH}$, AKR1B1, ABCG2, CA7, CA12, etc. Beta-sitosterol may have a high degree of binding with 19 targets such as AR, HMGCR, CYP51A1, etc. Sitosterol may have high binding degree with 46 targets such as AR, HMGCR, CYP51A1 and NPC1L1, while Stigmasterol may have high binding degree with 45 targets such as AR, NPC1L1, HMGCR, CYP51A1 and CYP19A1. Further details can be viewed in Supplementary File 1.

\section{Identification of Hub Targets of HCC}

GSE45436 was selected for this study, which was published on July 31, 2014, and the final data was updated on September 11, 2019. The GSE45436 dataset contains 134 samples, including 93 tumor samples and 41 normal control non-tumor samples. The expression profiles of 23,518 genes in normal samples $(n=41)$ and HCC samples $(n=93)$ were selected to identify the DEGs and construct gene coexpression networks. Four hundred and fifteen upregulated genes and 797 down-regulated genes were detected (Figure 2A). The top 50 up-regulated and downregulated DEGs were shown in the heatmap (Figure 2B). The power of $\beta=6$ (scale-free $R^{2}=0.85$ ) was selected as the soft-thresholding parameter to ensure a scale-free network (Figure 2C). Dynamic Tree Cut algorithm can identify the module, and the gene expression values in the module are in similarity. Highly similar modules were merged, and a total of 16 co-expression modules were identified. The modules contained at least 54 genes and 2056 genes at most, each module was marked with one color (Figure 2D). Module-trait relationships are shown in Figure 2E. Each row corresponds to a module eigengene, while each column corresponds to a clinical trait. The correlation coefficient (upper number) and corresponding $\mathrm{P}$-value (lower number) in each cell resulted in the correlation between the gene module and the clinical trait. Black module $(\mathrm{r}=-0.83, p=5 \mathrm{e}-35)$ shows the highest negative correlation between the normal samples and the HCC samples. Turquoise module $(\mathrm{r}=0.78, p=3 \mathrm{e}-28)$ shows the highest positive correlation between the normal samples and the HCC samples. Hence, the genes in black module and turquoise module can be regarded as the downregulated and up-regulated hub genes, respectively. The overlapping genes obtained by merging the DEGs in normal and tumor patients with turquoise and black modulerelated target genes could be recognized as the key disease-related genes. As shown in Figure 3A and B, a total of 454 key disease-related genes have been identified. Among all, 354 genes were up-regulated in HCC samples and 100 were down-regulated in HCC patients.

\section{Construction of the Potential Anti-HCC Target Genes of Scutellaria barbata}

By merging the target genes corresponding to the active components of Scutellaria barbata and the key diseaserelated genes, we found 21 common genes (CETP, CYP26A1, TYMS, NEK2, PARP1, AKR1C3, SQLE, NUAK1, AURKB, AURKA, POLA1, FEN1, KIF11, CHEK1, CDK1, NQO1, FABP5, IMPDH, TOP2A, AKR1B10, CDC25B) which were highly possible the key targets for Scutellaria barbata to anti-HCC (Figure 3C). However, only two genes were downregulated, other 19 genes were up-regulated in HCC patients. The 19 up-regulated targets were inputted into the STRING database to build a PPI network (Figure 4A). According to the MCC and DC, 10 critical nodes (TYMS, NEK2, AURKB, AURKA, POLA1, FEN1, KIF11, CHEK1, CDK1, TOP2A) were filtered as key targets for further molecular docking (Figure 4B). Active componentanti-hepatoma target-pathway network of Scutellaria barbata was built to elucidate intuitive interactions in Scutellaria barbata (Figure 5). As we cross verified these 10 critical target genes with the 16 approved components of Scutellaria barbata (Table 2), we found that AURKB and TOP2A were targeted by half of the components. MOL000098, MOL000351 and MOL012250 were the components of most target genes, while MOL001755 was predicted not to target any of the 10 critical target genes.

\section{GO and KEGG Enrichment Analysis}

The last 10 critical genes that were filtered as the key targets were selected to perform GO and KEGG enrichment analysis. According to $\mathrm{P}$ value $<0.05$, the top ten items of biological process (BP) of GO enrichment were mitotic centrosome separation, centrosome separation, centrosome cycle, histone phosphorylation, microtubule organizing center organization, regulation of mitotic nuclear division, regulation of centrosome cycle, nuclear division, regulation of nuclear division, organelle fission. And the top ten items of cellular component (CC) of GO analysis were chromosomal region, spindle microtubule, condensed chromosome, condensed nuclear chromosome, mitotic spindle, spindle, spindle pole, microtubule, 
A

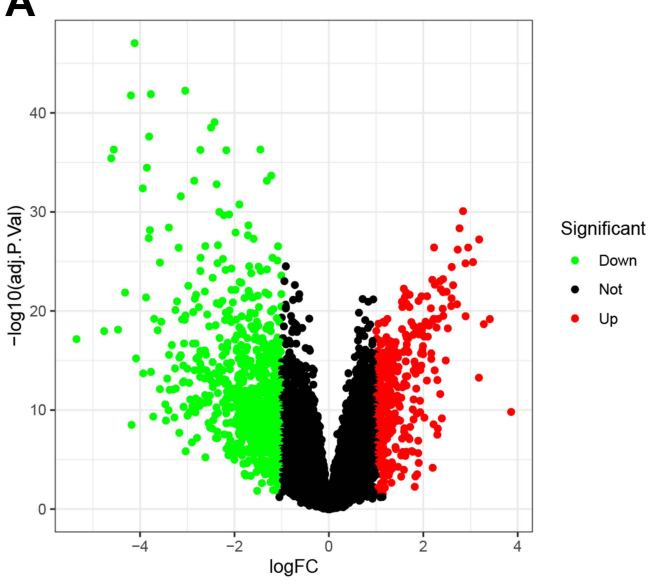

C

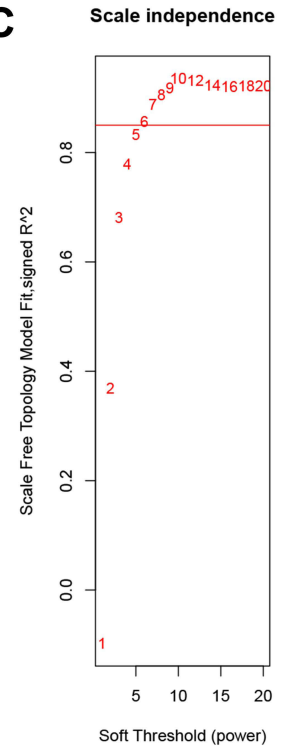

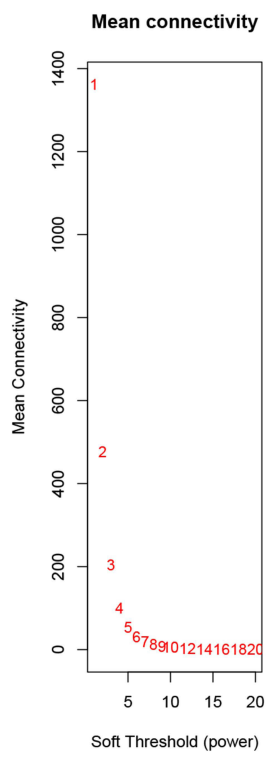

B

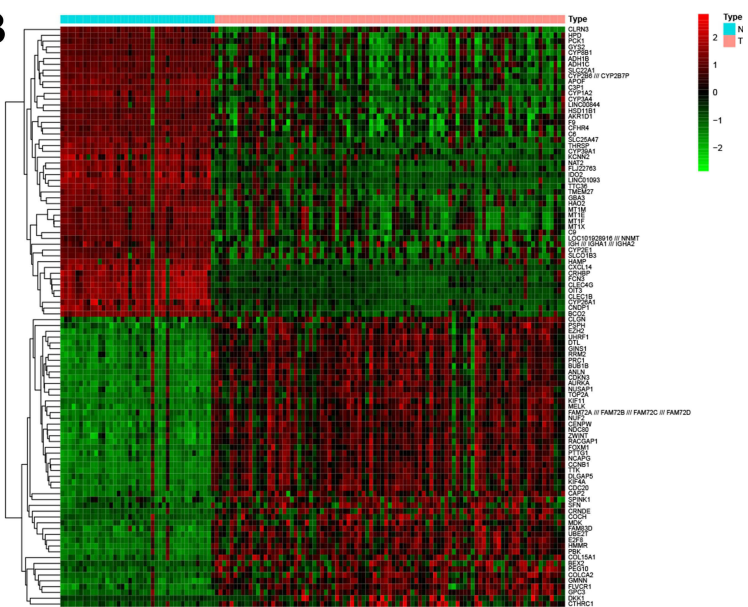

Cluster Dendrogram

D

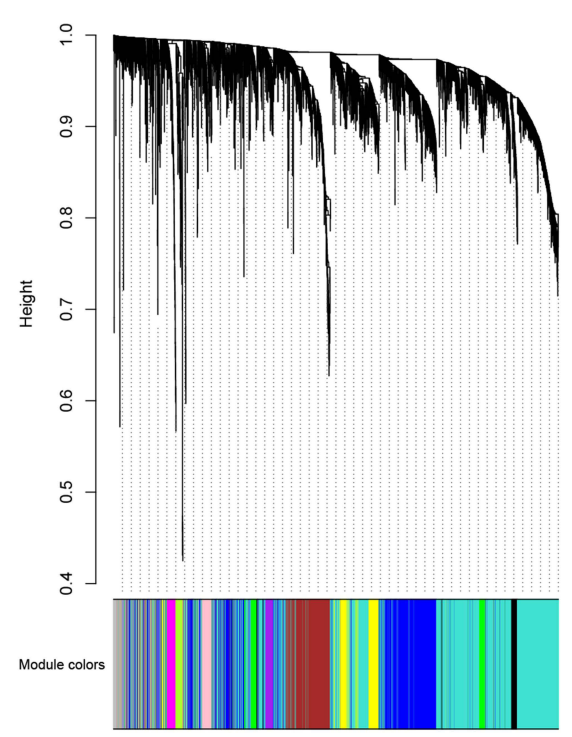

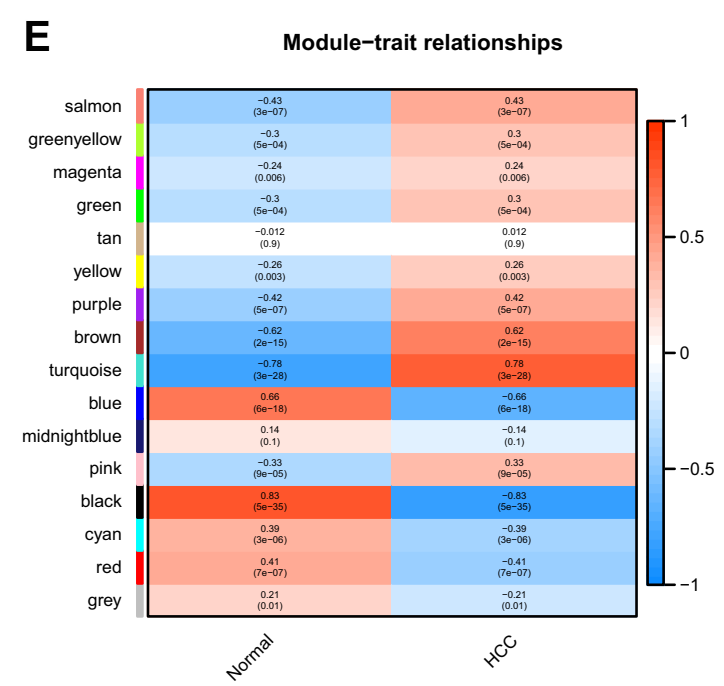

Figure 2 Identification of hub targets of HCC. (A) Volcano plot of DEGs, (B) Heatmap of the top 50 up-regulated and down-regulated DEGs, (C)Analysis of the scale-free network coefficient R-squared for the soft threshold, (D) A cluster dendrogram was built based on the dissimilarity of the topological overlap, (E) Module-trait relationships. 


\section{A}

\section{Turquoise Module}

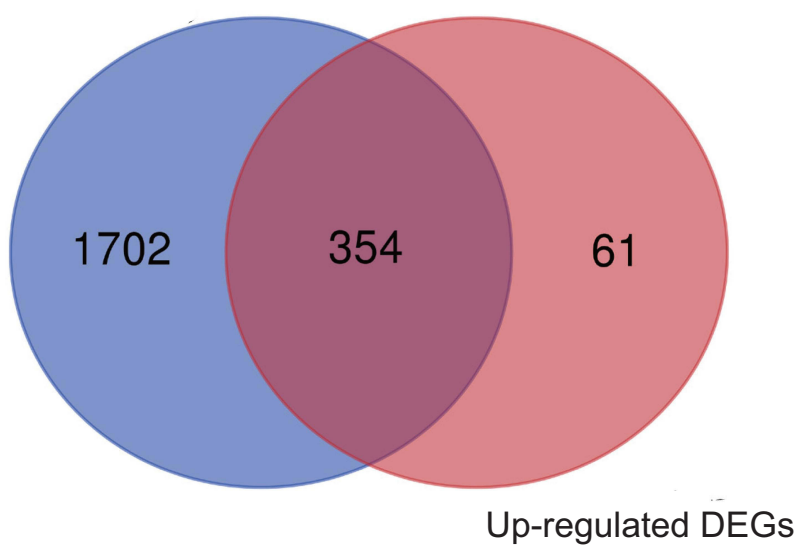

B

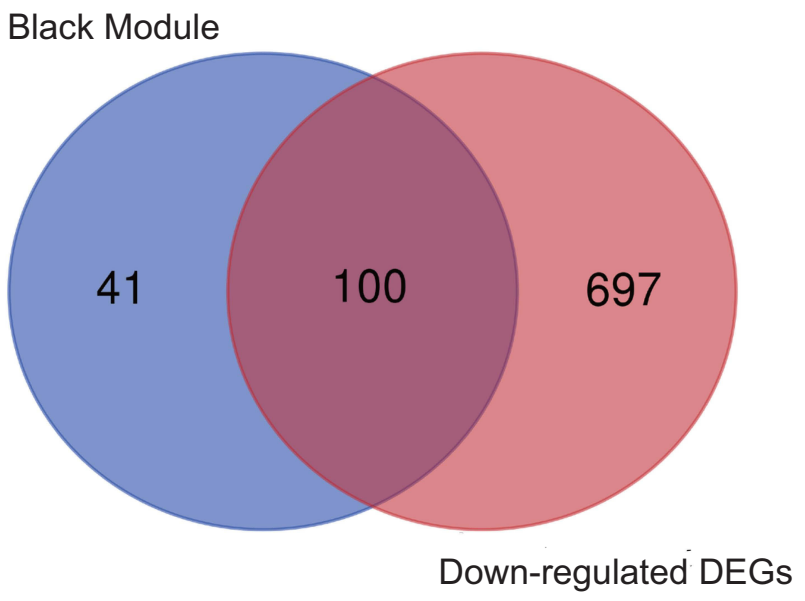

C

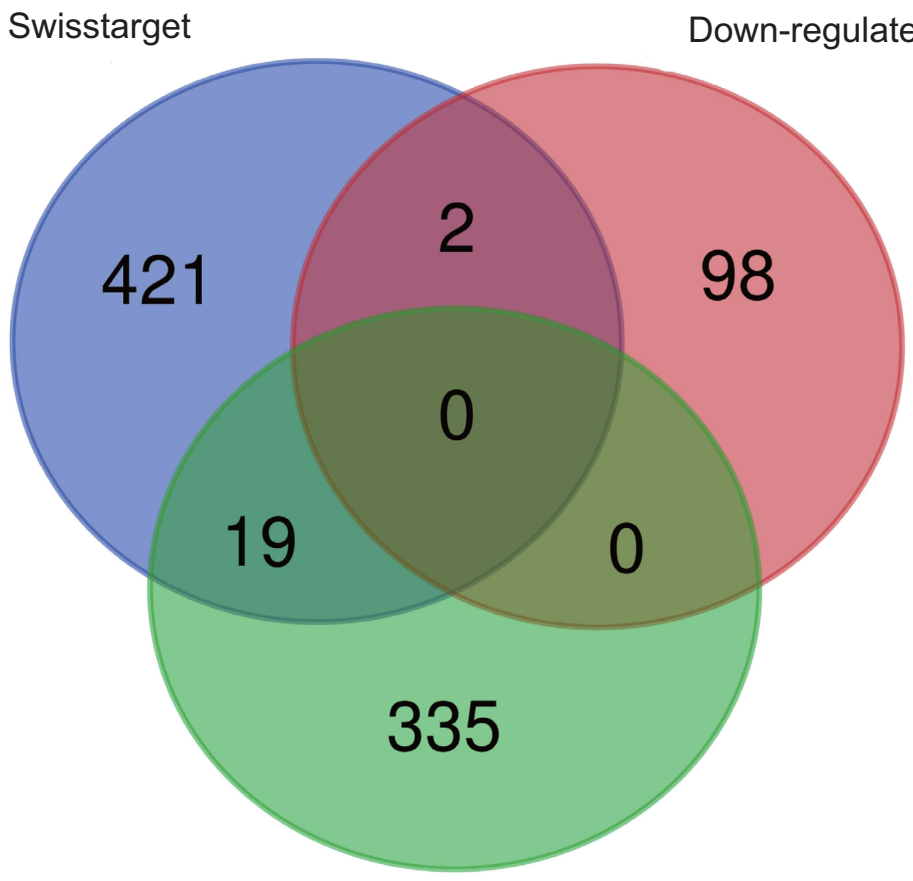

Up-regulated overplapping genes

Figure 3 Venn diagram. (A) Venn diagram of turquoise module and up-regulated DEGs, (B) Venn diagram of black module and down-regulated DEGs, (C) Venn diagram of up-regulated overlapping genes, down-regulated overlapping genes and SwisstargetPrediction targets.

midbody, spindle pole centrosome. The top ten items of molecular function (MF) of GO analysis were histone kinase activity, protein serine/threonine kinase activity, catalytic activity, acting on DNA, protein serine/threonine/tyrosine kinase activity, magnesium ion binding, RNA-DNA hybrid ribonuclease activity, folic acid binding, sequence-specific mRNA binding, translation repressor activity, mRNA regulatory element binding, endodeoxyribonuclease activity, producing 5'-phosphomonoesters (Figure 6A and B). The results of the GO analysis elucidated that Scutellaria barbata treatment of HCC was strongly associated with cell division pathway. According to the results of $\mathrm{R}$ package, a total of 13 pathways were screened by KEGG analysis, involving DNA 

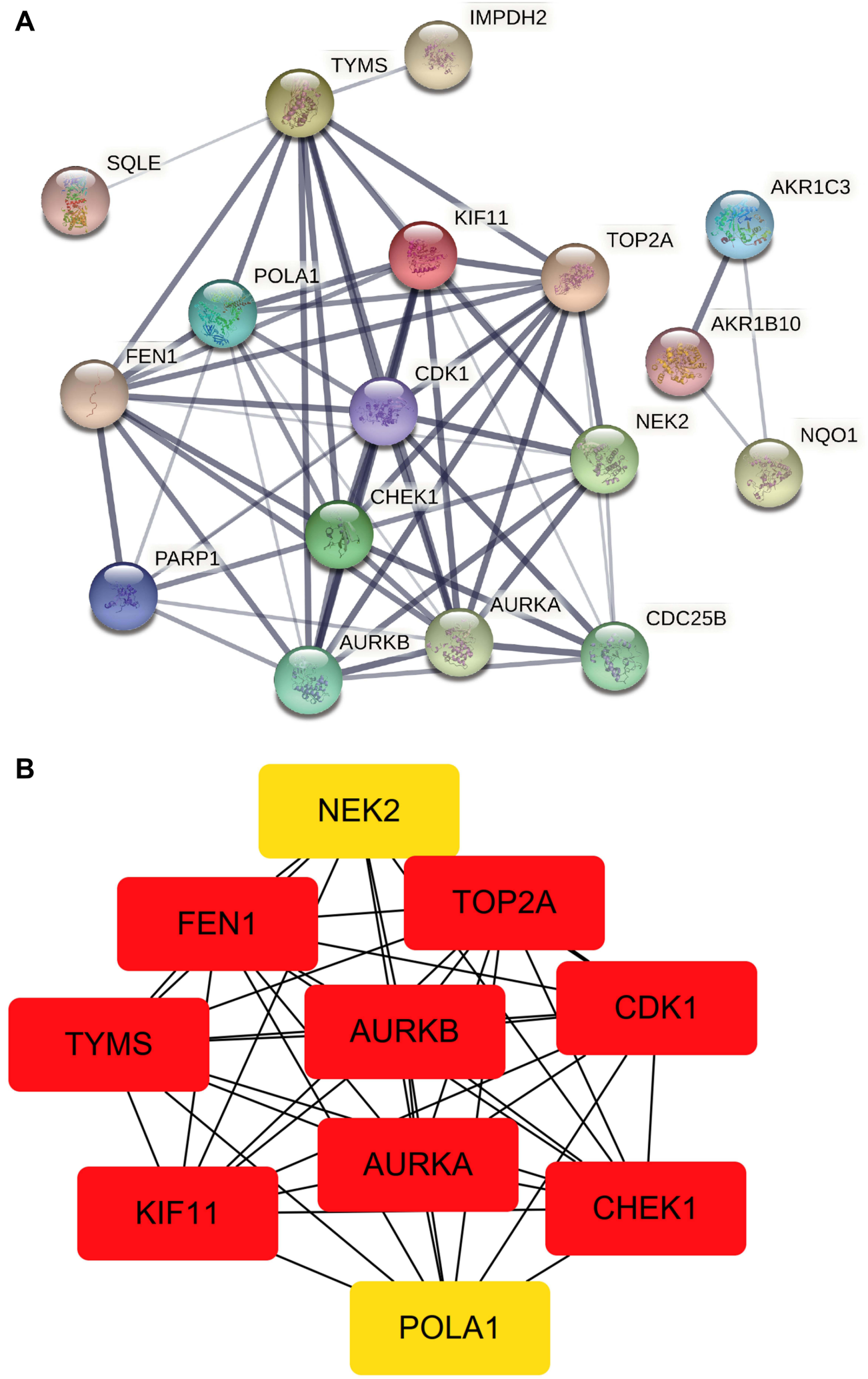

Figure 4 PPI analysis. (A) PPI network of the 19 up-regulated targets without disconnected nodes. (B) IOup-regulated targets that were screened according to the MCC and DC. 


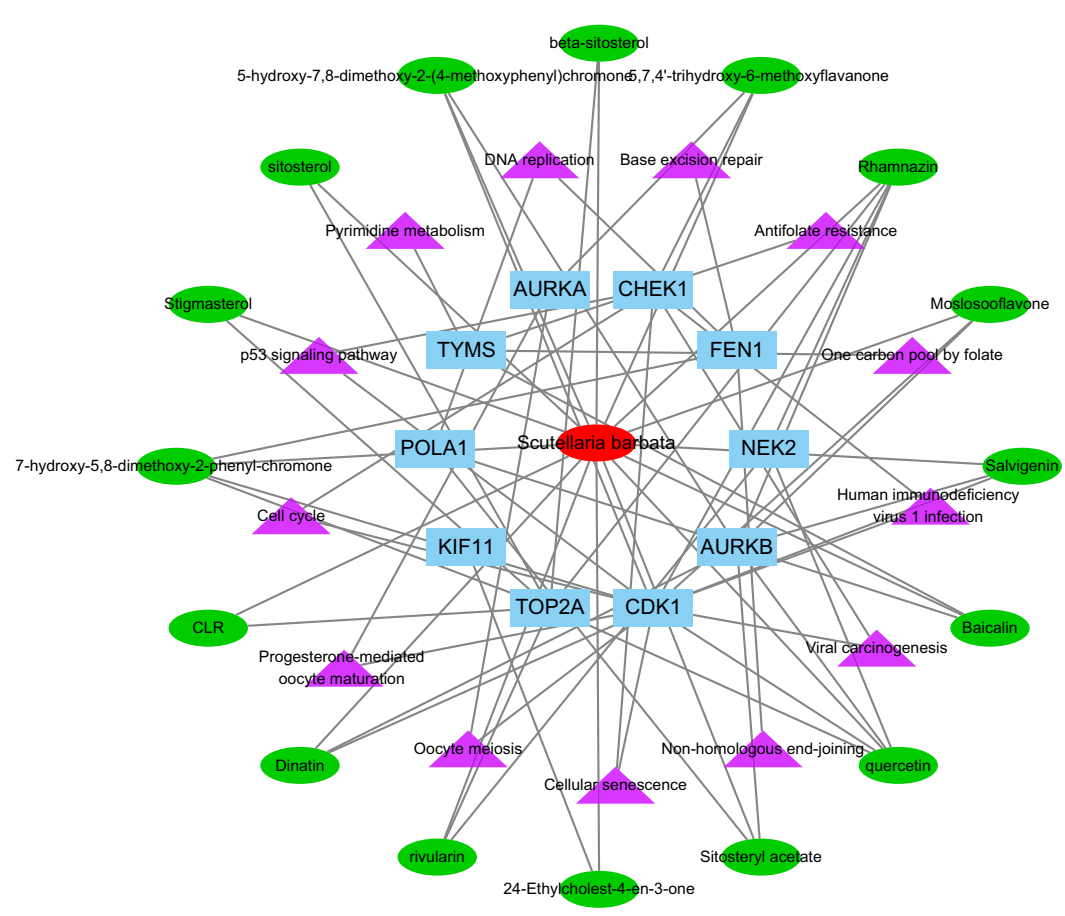

Figure 5 Active component-anti-hepatoma target-pathway network of Scutellaria barbata.

replication, p53 signaling pathway, Progesterone -mediated oocyte maturation, Cell cycle, Oocyte meiosis, Cellular senescence, Non-homologous end-joining, Viral carcinogenesis, Human immunodeficiency virus 1 infection, One carbon pool by folate, Antifolate resistance, Base excision repair, Pyrimidine metabolism (Figure 7A and B). The KEGG analysis via DAVID showed 5 pathways were involved, including Cell cycle, DNA replication, p53 signaling pathway, Pyrimidine metabolism and Oocyte meiosis.

\section{Molecular Docking for Computer Virtual Verification}

The 10 key targets in Figures 4B and 16 core components screened from Scutellaria barbata were performed by molecular docking, the basic information of ligands and proteins are shown in Table 3 . When the lowest binding energy is less than-5.0 $\mathrm{kJ} \cdot \mathrm{mol}-1$, it indicates that the docking effect between molecular ligand and receptor protein is good, and the lower the binding energy is, the better the binding capacity between molecule and protein is. The results showed that the majority of the binding energy were less than $-5.0 \mathrm{~kJ} \cdot \mathrm{mol}-1$, which revealed benign docking effect between drug components and target proteins, except for the binding energy between MOL000953, MOL000358,
MOL001973, MOL000449 and MOL000359and AURKA, as well as the binding energy between MOL000953, MOL000358, MOL001973, MOL000449, MOL000359, MOL001755 and FEN1 (Figure 8A).

Among those with better binding energy, the binding energy of AURKB to MOL000953, MOL000358, MOL000499, MOL001755, MOL001973 and MOL000359were less than that of the original ligand. The binding energy of TOP2A to MOL002776, MOL000953, MOL000358, MOL000449, MOL001735, MOL000351, MOL000359 and MOL000098 were less than that of the original ligand. The binding energy of CHEK1 to MOL002776 and MOL001755 were less than that of the original ligand. The binding energy of TYMS to MOL012250, MOL002776, MOL000953, MOL000358, MOL000449, MOL001973, MOL001755 and MOL000359 were less than that of the original ligand. The binding energy of NEK2 to MOL002776, MOL000358, MOL000098, MOL000449, MOL008206, MOL001755, MOL000351 and MOL000359 were less than that of the original ligand. The binding energy of POLA1 to all of the drug components except MOL001973 were less than that of the original ligand. The results above all showed that these target proteins had better ability to combine with the components of Scutellaria barbata, thus these target proteins may be the potential antiHCC mechanism of Scutellaria barbata. Further, based on 
Table 2 Cross Verification of the 10 Critical Target Genes and Compounds of Scutellaria barbata

\begin{tabular}{|c|c|c|}
\hline No. & $\begin{array}{l}\text { Components in } \\
\text { Scutellaria barbata }\end{array}$ & $\begin{array}{r}\text { Critical Targets Predicted } \\
\text { in Swiss Target Prediction } \\
\text { Database }\end{array}$ \\
\hline 1 & $\begin{array}{l}5,7,4^{\prime} \text {-trihydroxy } \\
-6 \text {-methoxyflavanone }\end{array}$ & AURKA, CHEKI, CHEKI \\
\hline 2 & $\begin{array}{l}\text { 5-hydroxy-7,8-dimethoxy- } \\
\text { 2-(4-methoxyphenyl) } \\
\text { chromone }\end{array}$ & AURKB, CDKI \\
\hline 3 & $\begin{array}{l}\text { 7-hydroxy-5,8-dimethoxy- } \\
\text { 2-phenyl-chromone }\end{array}$ & $\begin{array}{r}\text { NEK2, AURKB, FENI, CDKI, } \\
\text { TOP2A }\end{array}$ \\
\hline 4 & Baicalin & TYMS, POLAI \\
\hline 5 & CLR & TOP2A \\
\hline 6 & Beta-sitosterol & TOP2A \\
\hline 7 & Rivularin & NEK2, TOP2A \\
\hline 8 & Sitosteryl acetate & AURKB, POLAI \\
\hline 9 & Stigmasterol & TOP2A \\
\hline 10 & Dinatin & NEK2, AURKB, CDKI \\
\hline II & 24-Ethylcholest-4-en-3-one & I \\
\hline 12 & Salvigenin & AURKB, CDKI \\
\hline 13 & Rhamnazin & $\begin{array}{r}\text { NEK2, AURKB, CDKI, } \\
\text { TOP2A }\end{array}$ \\
\hline 14 & Sitosterol & TOP2A \\
\hline 15 & Moslosooflavone & AURKB, CDKI \\
\hline 16 & Quercetin & $\begin{array}{r}\text { NEK2, AURKB, CDKI, } \\
\text { TOP2A }\end{array}$ \\
\hline
\end{tabular}

binding energy and molecular interactions such as hydrogen bonds, we selected the top 4 potential combinations with the best binding energy and molecular interactions. Finally, we found the following combinations, including MOL000449 and AURKB (Figure 8B), MOL002776 and CHEK1 (Figure 8C), MOL002776 and NEK2 (Figure 8D), MOL000098 and CHEK1 (Figure 8E). AURKB, CHEK1 and NEK2 could be the top 3 potential target proteins of Scutellaria barbata in treating HCC.

\section{Prognostic Value of AURKB, CHEKI and NEK2}

As we considered that AURKB, CHEK1 and NEK2 could be the top 3 potential target proteins of Scutellaria barbata in treating HCC. We further explored the prognostic value of AURKB, CHEK1 and NEK2 in HCC. As shown in Figure 9, high expression of AURKB, CHEK1 and NEK2 were consistently linked to poor prognosis of OS and DFS. High expression of the AURKB was related to poor prognosis for HCC in the aspect of OS (Figure 9A, $\mathrm{P}=0.029$ ) and DFS (Figure 9B, $\mathrm{P}=0.048$ ). High expression of the CHEK1 was related to poor prognosis for $\mathrm{HCC}$ in the aspect of $\mathrm{OS}$ (Figure 9C, $\mathrm{P}<0.001$ ) and DFS (Figure 9D, $\mathrm{P}<0.001$ ). High expression of the NEK2 was related to poor prognosis for $\mathrm{HCC}$ in the aspect of OS (Figure 9E, $\mathrm{P}<0.001$ ) and DFS (Figure 9F, $\mathrm{P}<0.001$ ).

\section{Discussion}

Scutellaria barbata is a plant of Scutellaria in Labiatae, which has the functions of clearing heat and detoxification, promoting blood circulation and removing blood stasis, reducing swelling and relieving pain in TCM. A large number of modern pharmacological studies have confirmed that Scutellaria barbata had anti-tumor effect. Zhang et al showed that Scutellaria barbata could inhibit the proliferation of A2780 cells and induce apoptosis through mitochondrial pathway, thus playing the role of anti-ovarian cancer. ${ }^{20}$ Dai et al revealed that Scutellaria barbata inhibited invasion of HCC via MMP/TIMP in vitro. ${ }^{21}$ Scutellaria barbata polysaccharides inhibited the growth of HCC in mice and affected serum proteomic profiling. ${ }^{6}$ Extraction of Scutellaria barbata inhibited the tumor growth through downregulation of Treg cells and manipulating Th1/Th17 immune response in hepatoma H22-bearing mice. ${ }^{22}$

Although Gong et al have performed a network pharmacology to explore the mechanism of Scutellaria barbata for treating $\mathrm{HCC}$, and they concluded that Scutellaria barbata exerted its anti-hepatoma effect by targeting

Table 3 Information of Proteins Involved in Molecular Docking

\begin{tabular}{|l|c|c|c|}
\hline No. & Target Protiens & PDB-ID & Ligand-ID \\
\hline 1 & CDKI & 3 LFS & A07 \\
2 & AURKB & 4 AF3 & VX6 \\
3 & TOP2A & IZXN & ADP \\
4 & CHEKI & $4 Q Y E$ & $3 D L$ \\
5 & AURKA & 5 DR6 & $5 E I$ \\
6 & KIFII & 3 LH & EMQ \\
7 & FENI & 6 CVR & AMP \\
8 & TYMS & $5 X 66$ & MTX \\
9 & NEK2 & $2 X K 8$ & $5 R I$ \\
10 & POLAI & $4 Q 5 V$ & $2 Z E$ \\
\hline
\end{tabular}




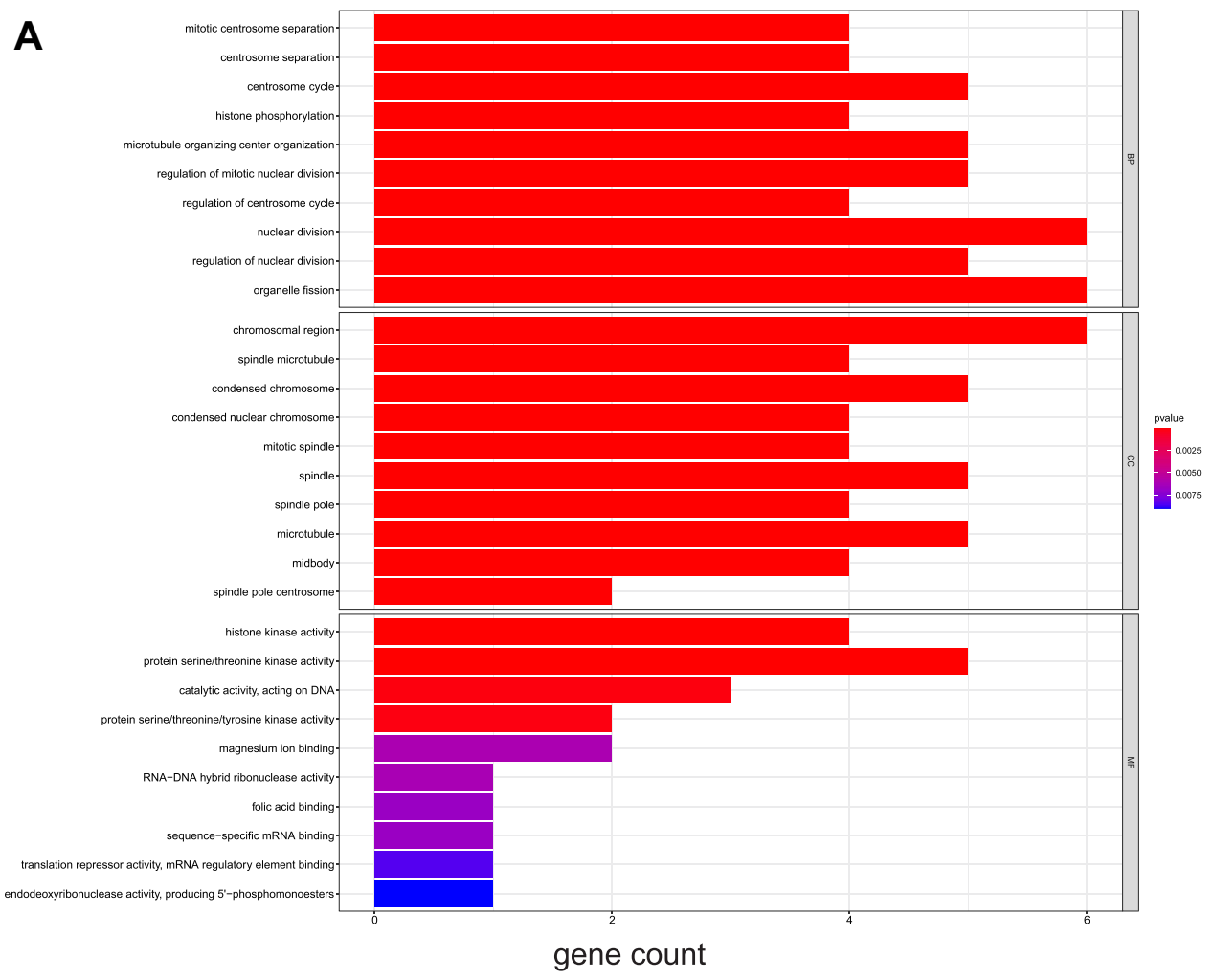

B

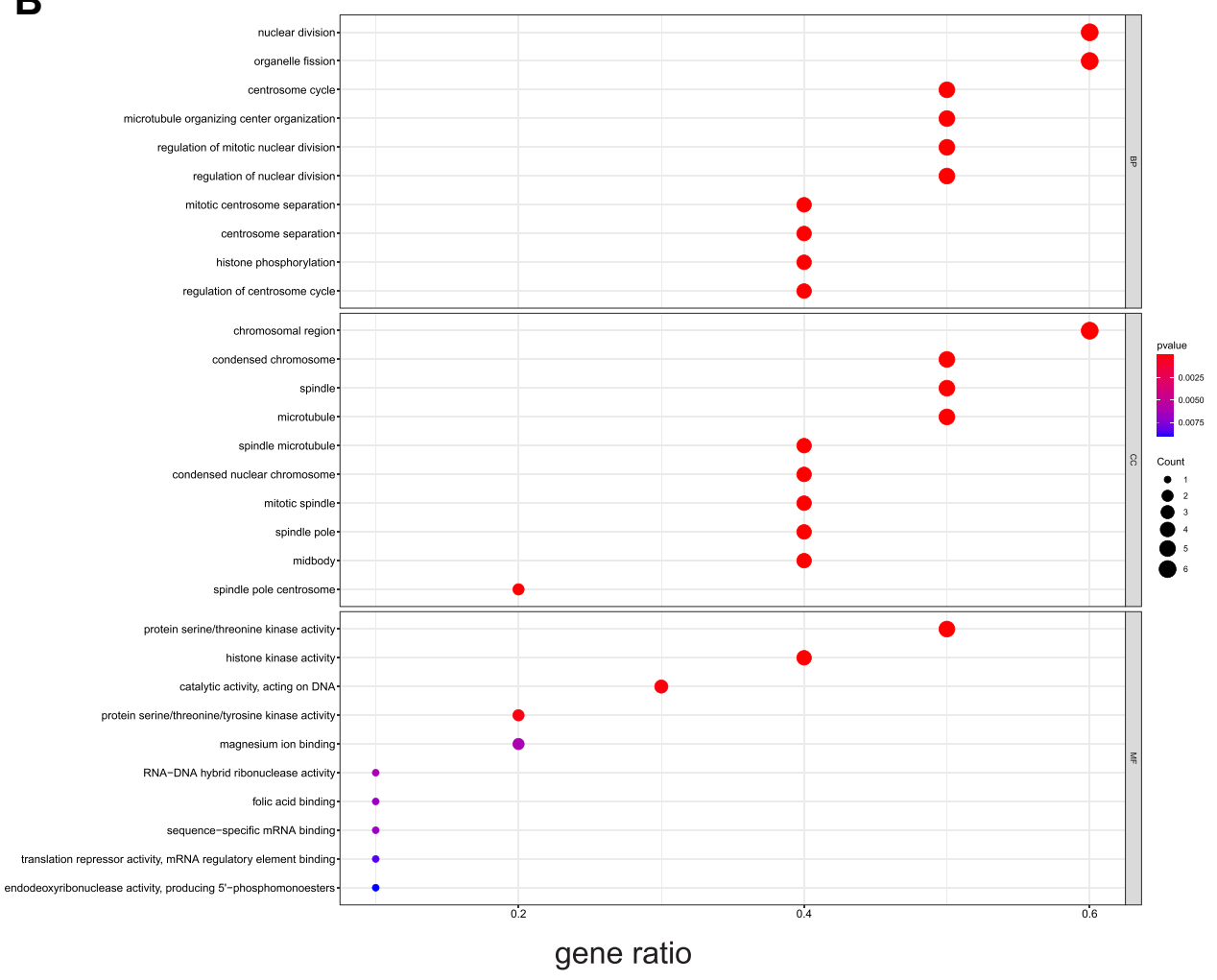

Figure 6 The results of GO enrichment analysis. (A) The barplot of GO enrichment analysis, (B) The bubble plot of GO enrichment analysis. "Gene ratio" means the percentage of the genes that enriched in corresponding pathway in all the genes that annotated in GO dataset. 
A

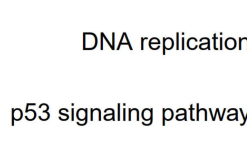

Progesterone-mediated oocyte maturation -

Cell cycle-

Oocyte meiosis -

Cellular senescence

Non-homologous end-joining -

Viral carcinogenesis

Human immunodeficiency virus 1 infection-

One carbon pool by folate

Antifolate resistance

Base excision repair

Pyrimidine metabolism -
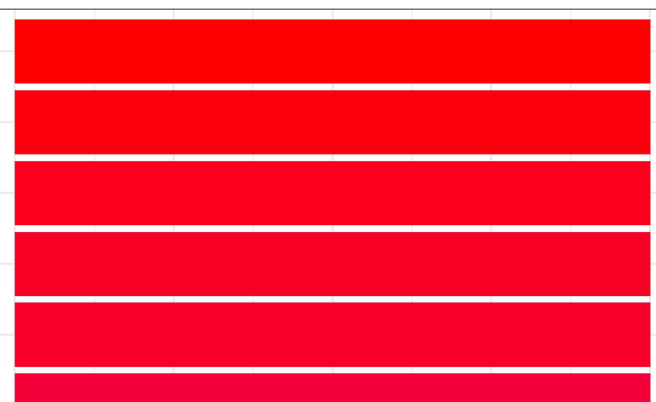

pvalue
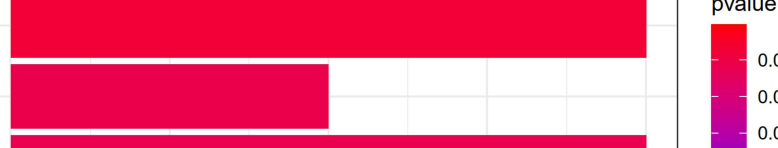

$-0.01$

$-0.02$
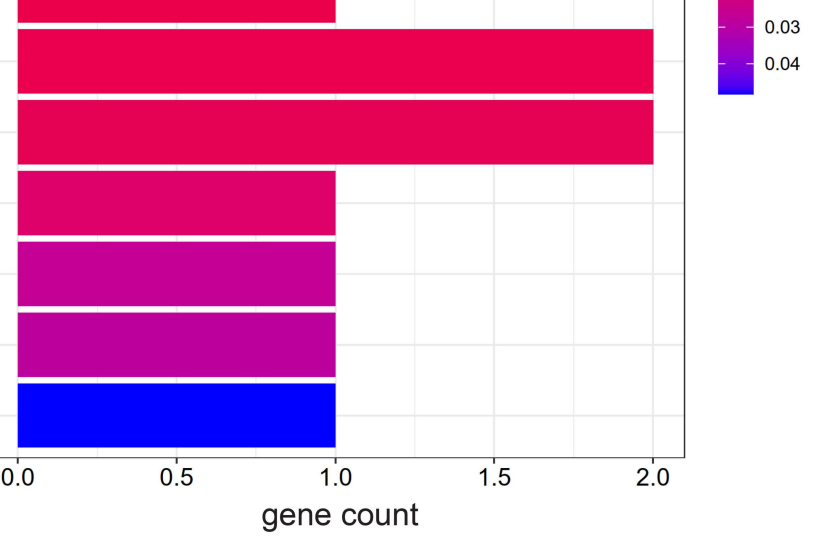

B

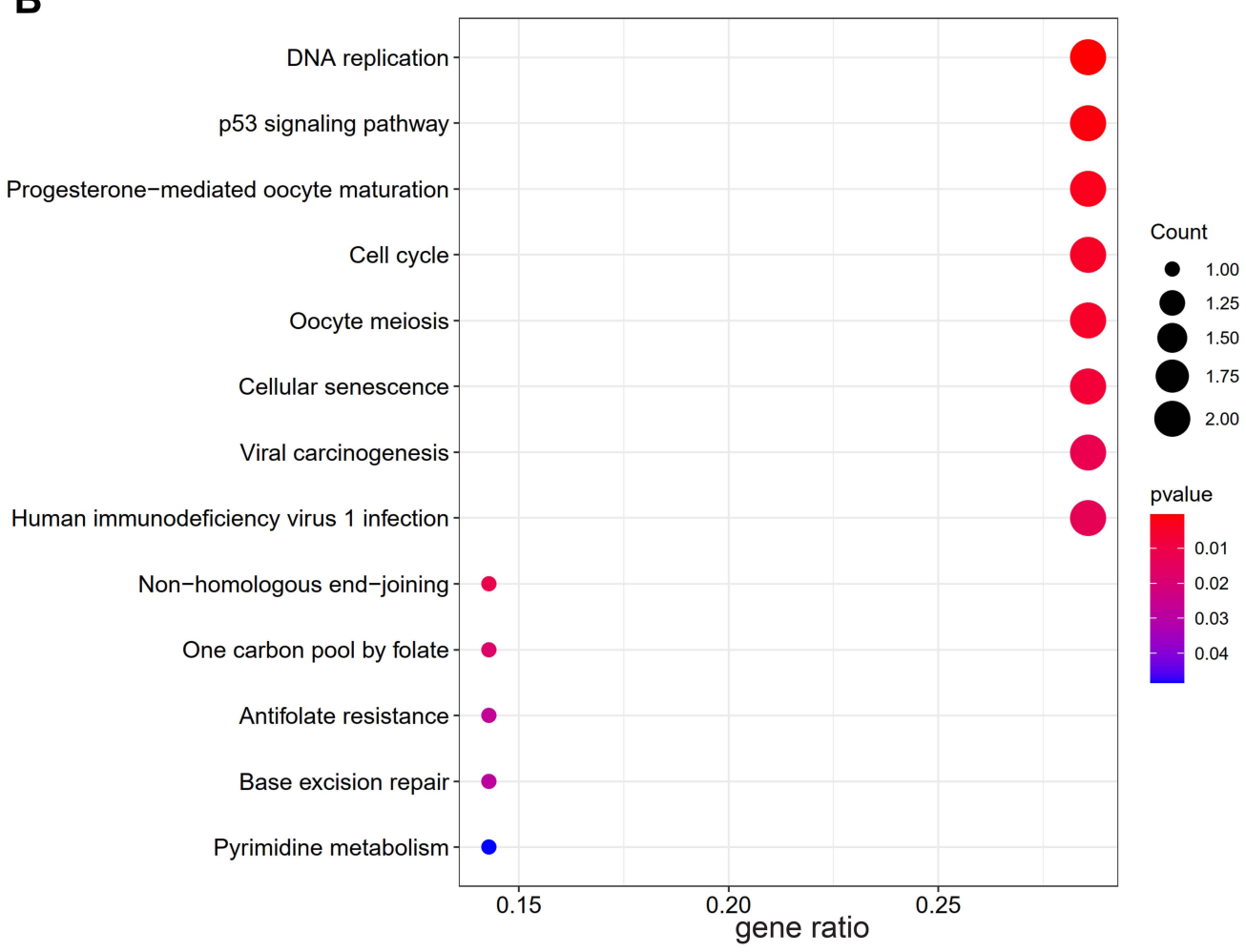

Figure 7 The results of KEGG pathways enrichment analysis. (A) The barplot of KEGG analysis, (B) The bubble plot of KEGG analysis. "Gene ratio" means the percentage of the genes that enriched in corresponding pathway in all the genes that annotated in GO dataset. 
A

\begin{tabular}{|c|c|c|c|c|c|c|c|c|c|c|}
\hline MOL012245 & -7.4 & -8.4 & -8.1 & -8.8 & -7.5 & -7.6 & -7.7 & -7.8 & -7.9 & -8.1 \\
\hline MOL012248 & -7.7 & -7.9 & -7.7 & -8.9 & -8.1 & -6.6 & -8.6 & -7.6 & -8.3 & -7.6 \\
\hline MOL012250 & -8.0 & -8.4 & -7.8 & -8.4 & -7.0 & -8.3 & -8.3 & -8.1 & -7.9 & -8.2 \\
\hline MOL002776 & -7.1 & -7.7 & -8.7 & -9.2 & -7.7 & -8.4 & -5.4 & -8.0 & -9.2 & -8.1 \\
\hline MOL000953 & -7.8 & -10.4 & -8.3 & -8.1 & -3.5 & -8.5 & -2.3 & -8.8 & -7.3 & -7.7 \\
\hline MOL000358 & -8.5 & -9.9 & -8.4 & -8.3 & -4.9 & -7.9 & -3.5 & -8.8 & -8.6 & -7.9 \\
\hline MOL012266 & -7.8 & -7.5 & -7.5 & -9.0 & -7.2 & -7.8 & -7.7 & -7.2 & -8.2 & -8.3 \\
\hline MOL001973 & -8.5 & -9.3 & -8.0 & -8.5 & -3.5 & -7.7 & -1.1 & -8.9 & -7.3 & -6.5 \\
\hline MOL000449 & -7.8 & -10.0 & -8.7 & -8.3 & -2.6 & -9.0 & -2.6 & -9.4 & -8.7 & -8.2 \\
\hline MOL001735 & -7.7 & -8.4 & -8.3 & -9.0 & -8.0 & -7.6 & -7.8 & -7.7 & -8.1 & -8.0 \\
\hline MOL001755 & -8.6 & -10.0 & -8.1 & -9.4 & -5.1 & -8.1 & -3.5 & -9.1 & -8.4 & -8.1 \\
\hline MOL002915 & -7.4 & -8.1 & -8.0 & -8.5 & -7.9 & -6.7 & -8.3 & -7.9 & -7.2 & -7.1 \\
\hline MOL000351 & -7.6 & -8.0 & -8.3 & -8.8 & -7.4 & -7.1 & -7.7 & -7.7 & -9.1 & -7.8 \\
\hline MOL000359 & -8.1 & -10.0 & -8.8 & -8.3 & -4.7 & -7.9 & -3.5 & -9.2 & -8.4 & -7.5 \\
\hline MOL008206 & -7.8 & -7.9 & -7.9 & -8.8 & -8.1 & -8.1 & -8.4 & -7.3 & -8.9 & -8.6 \\
\hline MOL000098 & -7.9 & -8.1 & -8.9 & -8.9 & -7.5 & -8.4 & -8.4 & -7.5 & -8.8 & -8.6 \\
\hline \multirow[t]{2}{*}{ Legand } & -8.8 & -9.2 & -8.1 & -9.0 & -8.4 & -10.1 & -10.0 & -7.9 & -8.3 & -6.8 \\
\hline & CDK1 & AURKB & TOP2A & CHEK1 & AURKA & KIF11 & FEN1 & TYMS & NEK2 & POLA1 \\
\hline
\end{tabular}

B

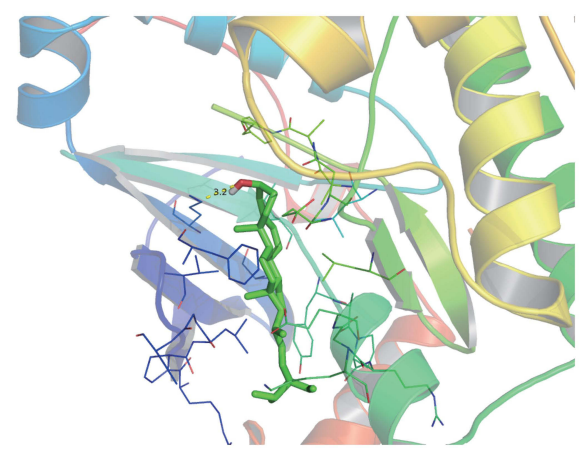

D

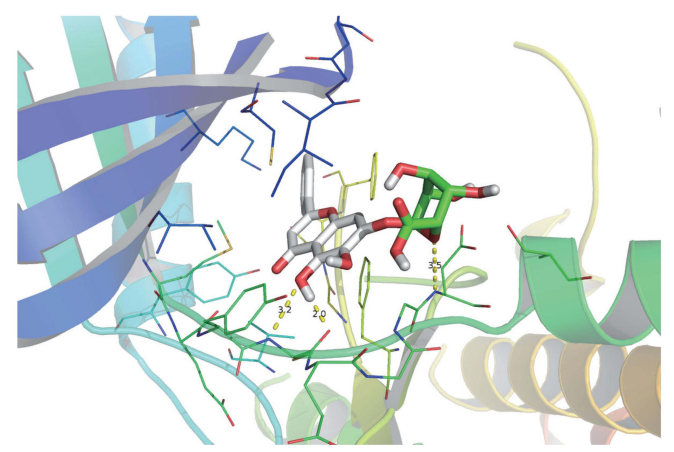

C

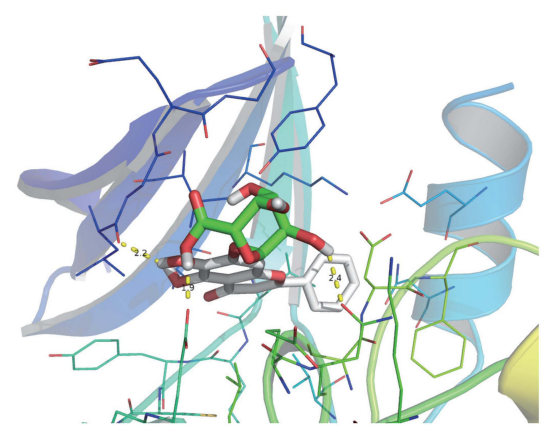

$\mathbf{E}$

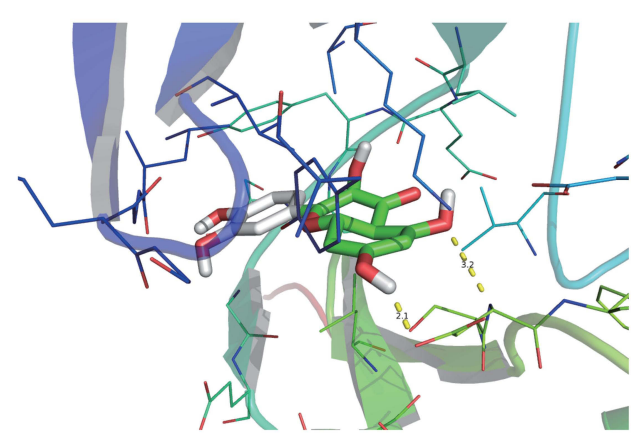

Figure 8 The results of molecular docking. (A) Heatmap of binding energy between active components and target proteins, (B) Visualization of molecular docking between MOL000449 and AURKB, (C) Visualization of molecular docking between MOL002776 and CHEKI, (D) Visualization of molecular docking between MOL002776 and NEK2, (E) Visualization of molecular docking between MOL000098 and CHEKI. 
A

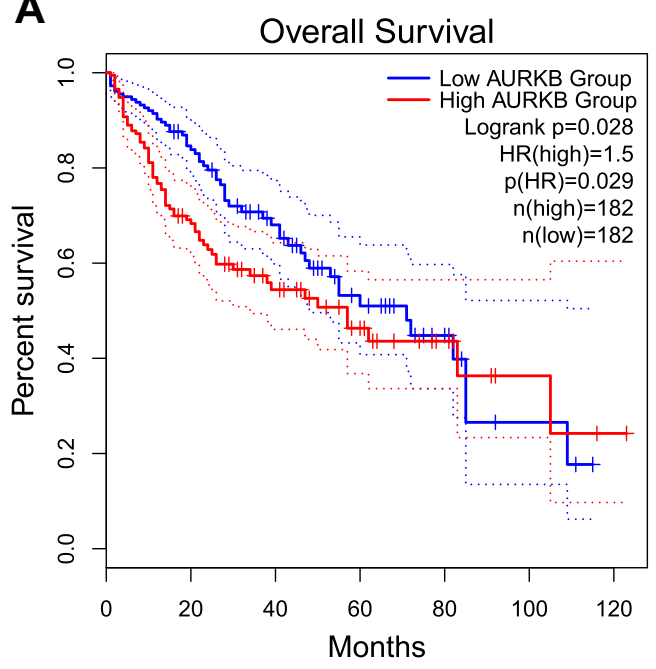

C

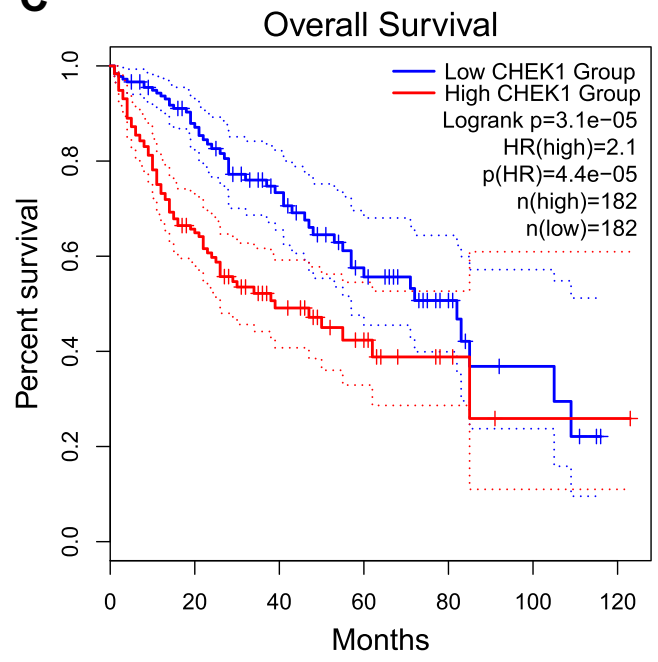

E

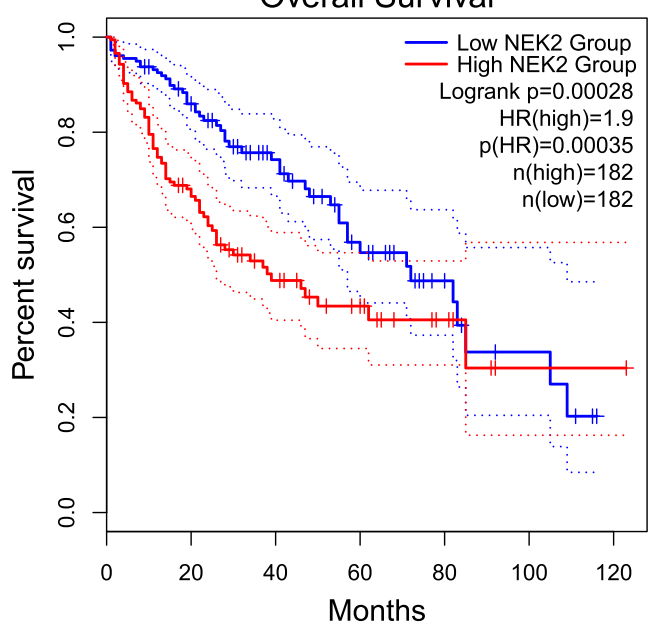

B

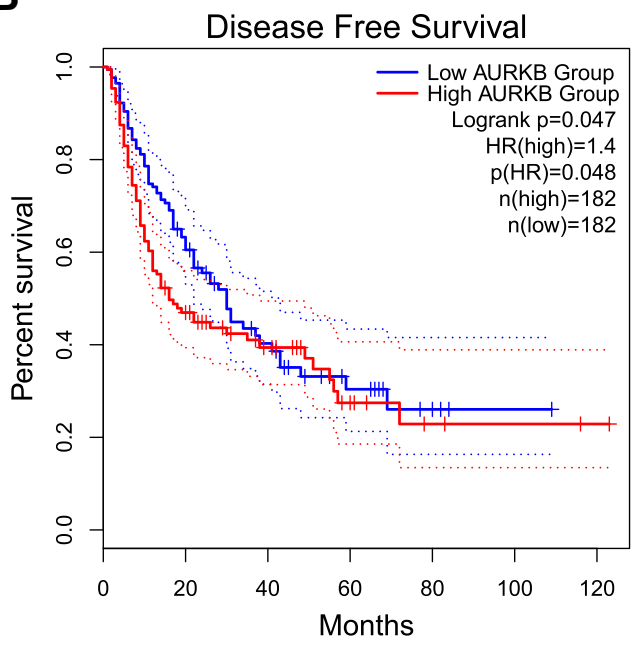

D

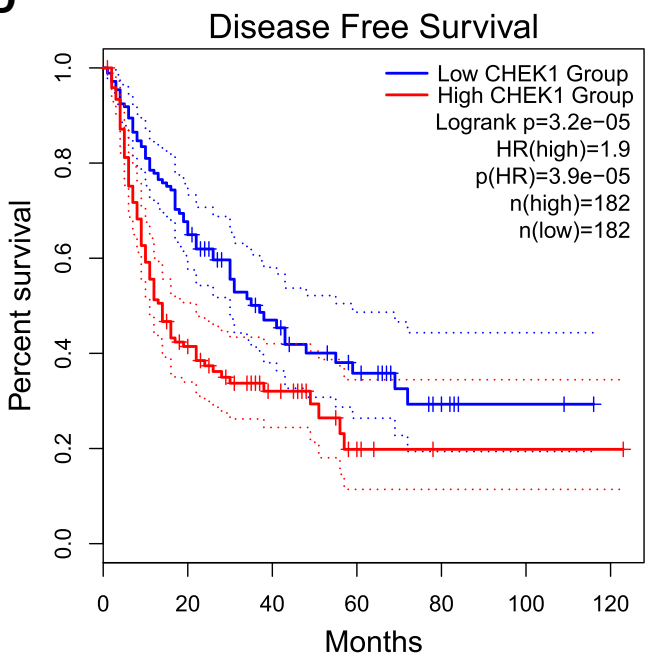

$\mathbf{F}$

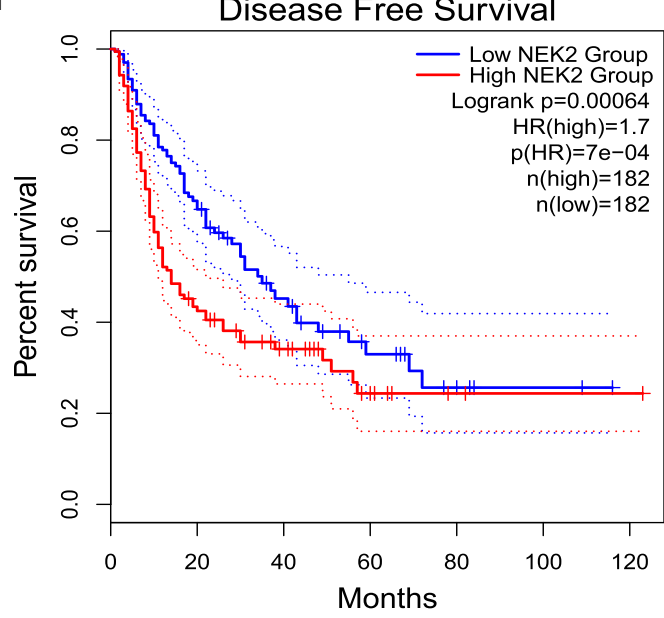

Figure 9 The results of Prognostic value of AURKB, CHEKI and NEK2. (A) Overall Survival Kaplan-Meier curve of AURKB, (B) Disease Free Survival Kaplan-Meier curve of AURKB, (C) Overall Survival Kaplan-Meier curve of CHEKI, (D) Disease Free Survival Kaplan-Meier curve of CHEKI, (E) Overall Survival Kaplan-Meier curve of NEK2, (F) Disease Free Survival Kaplan-Meier curve of NEK2. 
TTR through neutrophil degranulation pathway, ${ }^{23}$ our study combined network pharmacology and bioinformatics analysis in order to provide a more specific anti-HCC mechanism of Scutellaria barbata. Besides, we further investigated the reliability of our target prediction through computer virtual verification.

In this study, we recognized 10 critical target genes as the potential anti-HCC mechanism of Scutellaria barbata through bioinformatics analysis, namely TYMS, NEK2, AURKB, AURKA, POLA1, FEN1, KIF11, CHEK1, CDK1, TOP2A. These genes were all significantly overexpressed in HCC patients and altogether involved in cell cycle, DNA replication, p53 signaling pathway and Oocyte meiosis. To be more accurate, we discovered that AURKB, CHEK1 and NEK2 could be the top 3 potential target proteins of Scutellaria barbata in treating HCC based on binding energy and molecular interactions such as hydrogen bonds. AURKB is considered to be an abnormally expressed gene in many cancer and tumor cell lines. $^{24-28}$ AURKB overexpression is an independent molecular marker predicting tumor invasiveness and poor prognosis of HCC. ${ }^{29}$ AURKB has several functions during mitosis, including spindle assembly, chromosome segregation, and cytokinesis. ${ }^{30}$ As cells enter mitosis, survivin recruits AURKB to the inner centromere. ${ }^{31}$ It has been reported that AURKB together with passengers inner centromere protein (INCENP) and surviving constituted chromosomal passengers, which binds to the internal

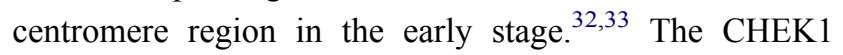
kinase is mainly responding to single-strand breaks, and overexpression of CHEK1 in germinal vesicle oocytes could arrest oocytes at the germinal vesicle stage. ${ }^{34}$ CHEK1 is responsible for controlling G1/S, S, and G2/M checkpoints. $^{35}$ One study demonstrated the LINC01224/ miR-330-5p/CHEK1 axis was involved in the pathology of HCC. $^{36}$ Bao et al highlighted that PLK-4/ATR/CHEK1 pathway might be a potential therapeutic target for HCC. ${ }^{37} \mathrm{NEK} 2$ is a serine/threonine centrosomal kinase, which is highly expressed and activated in S and G2 phases of the cell cycle. ${ }^{38}$ NEK2 plays a crucial role in cell cycle progression in the inter- and M-phases. ${ }^{39}$ Zhang et al have found that NEK2 could promote the metastasis of HCC. ${ }^{40}$ Numerous studies have shown that NEK2 could mediate tumor growth, metastasis and angiogenesis through mitosis. ${ }^{39,41-44} \mathrm{Wu}$ et al suggested that NEK2 modulated the growth, drug resistance, metastasis and angiogenesis of hepatoma cell via $\mathrm{pAKT} / \mathrm{NF}-\kappa \mathrm{B}$ signaling and matrix metalloproteinase activation. ${ }^{45}$ According to the aforementioned content, the results that AURKB, CHEK1 and NEK2 were abnormally escalated in HCC patients showed in our study were consistent with the previous publication. In the result of molecular docking and the binding energy value, we speculate that Scutellaria barbata exhibits anti-HCC role by inhibiting the function of these protein via protein-protein interaction.

\section{Conclusion}

In summary, our study suggests that Scutellaria barbata plays a key role in the treatment of $\mathrm{HCC}$ with multiple targets and pathways. AURKB, CHEK1 and NEK2 could be the potential target proteins of Scutellaria barbata in treating HCC. Cell cycle, DNA replication, p53 signaling pathway and Oocyte meiosis consist of the fundamental regulation cores in this mechanism.

\section{Abbreviations}

HCC, Hepatocellular carcinoma; TCMSP, Traditional Chinese Medicine Systems Pharmacology; STRING, search tool for recurring instances of neighbouring genes; DL, Drug-likeness; OB, Oral bioavailability; GEO, Gene Expression Omnibus; WGCNA, Weighted gene co-expression network analysis; PPI, protein-protein interaction network; DAVID, Database for Annotation, Visualization and Integrated Discovery; GO, Gene ontology; KEGG, Kyoto Encyclopedia of Genes and Genomes; HBV, Hepatitis B virus; TCM, Traditional Chinese medicine; DEGs, differentially expressed genes; CSV, comma-separated value; FC, fold change; TSV, tab-separated value; DC, degree centrality; EPC, Edge Percolated Component; MNC, Maximum Neighborhood Component; DMNC, Density of Maximum Neighborhood Component; MCC, Maximal Clique Centrality; BN, Bottleneck; CC, closeness centrality; BC, betweenness centrality; PDB, Protein Data Bank; BP, Biological process; CC, cellular component; MF, molecular function; INCENP, passengers inner centromere protein; AURKB, Serine/threonine-protein kinase Aurora-B; CDK1, Cyclin-dependent kinase 1; NEK2, Serine/threonine-protein kinase NEK2; FEN1, Flap endonuclease 1; TOP2A, DNA topoisomerase II alpha; AURKA, Serine/threonine-protein kinase AuroraA; CHEK1, Serine/threonine-protein kinase Chk1; TYMS, thymidylate synthetase; KIF11, kinesin family member 11; POLA1, DNA polymerase alpha 1, catalytic subunit. 


\section{Data Sharing Statement}

All data are available in the manuscript and they are shown in figures and tables.

\section{Acknowledgments}

The authors would like to thank the reviewers and also the authors of all references. The reviewer's advice really makes the great improvement of this paper.

\section{Author Contributions}

All authors made substantial contributions to conception and design, acquisition of data, or analysis and interpretation of data; took part in drafting the article or revising it critically for important intellectual content; agreed to submit to the current journal; gave final approval of the version to be published; and agree to be accountable for all aspects of the work.

\section{Funding}

This work was supported by National Natural Science Foundation of China (81873303), Natural Science Foundation of Guangdong Province, China (2019A1515011013), Key Projects of Educational Commission of Guangdong Province, China (2019KZDXM045), Administration of Traditional Chinese Medicine of Guangdong Province, China (20211122), and Medical innovation project of the First Affiliated Hospital of Guangzhou University of Chinese Medicine (2019IIT18), Guangzhou University of Chinese Medicine first-class discipline Construction Research Key Project (A1-AFD018181A27); Guangdong Famous Traditional Chinese Medicine Doctor Inheritance Studio of Fengbin Liu (Guangdong Traditional Chinese Medicine Office [2020] No. 1).

\section{Disclosure}

The authors declare that the research was conducted in the absence of any commercial or financial relationships that could be construed as a potential conflict of interest.

\section{References}

1. Gou Y, Yi J, Jiang M, Cao C. Analysis on effects of comprehensive nursing care applied in interventional therapy for patients with liver cirrhosis and liver cancer. Iran J Public Health. 2019;48(3):494-500.

2. Nagtegaal ID, Odze RD, Klimstra D, et al. The 2019 WHO classification of tumours of the digestive system. Histopathology. 2020;76 (2):182-188. doi:10.1111/his.13975
3. Craig AJ, von Felden J, Garcia-Lezana T, Sarcognato S, Villanueva A. Tumour evolution in hepatocellular carcinoma. Nat Rev Gastroenterol Hepatol. 2020;17(3):139-152. doi:10.1038/ s41575-019-0229-4

4. Sagnelli E, Macera M, Russo A, Coppola N, Sagnelli C. Epidemiological and etiological variations in hepatocellular carcinoma. Infection. 2020;48(1):7-17. doi:10.1007/s15010-01901345-y

5. Mandlik DS, Mandlik SK. Herbal and natural dietary products: upcoming therapeutic approach for prevention and treatment of hepatocellular carcinoma. Nutr Cancer. 2020;1-25. doi:10.1080/ 01635581.2020 .1834591

6. Li L, Xu X, Wu L, et al. Scutellaria barbata polysaccharides inhibit tumor growth and affect the serum proteomic profiling of hepatoma H22 bearing mice. Mol Med Rep. 2019;19(3):2254-2262. doi:10.3892/mmr.2019.9862

7. Ma TT, Zhang GL, Dai CF, et al. Scutellaria barbata and Hedyotis diffusa herb pair for breast cancer treatment: potential mechanism based on network pharmacology. $J$ Ethnopharmacol. 2020;259:112929. doi:10.1016/j.jep.2020.112929

8. Wang L, Xu J, Yan Y, et al. Green synthesis of gold nanoparticles from Scutellaria barbata and its anticancer activity in pancreatic cancer cell (PANC-1). Artif Cells Nanomed Biotechnol. 2019;47 (1):1617-1627. doi:10.1080/21691401.2019.1594862

9. Li H, Su J, Jiang J, et al. Characterization of polysaccharide from Scutellaria barbata and its antagonistic effect on the migration and invasion of HT-29 colorectal cancer cells induced by TGF-beta1. Int J Biol Macromol. 2019;131(48):886-895. doi:10.1016/j. ijbiomac.2019.03.053

10. Dai ZJ, Wu WY, Kang HF, et al. Protective effects of Scutellaria barbata against rat liver tumorigenesis. Asian Pac J Cancer Prev. 2013;14(1):261-265. doi:10.7314/APJCP.2013.14.1.261

11. Hopkins AL. Network pharmacology: the next paradigm in drug discovery. Nat Chem Biol. 2008;4(11):682-690. doi:10.1038/ nchembio. 118

12. Xing $\mathrm{S}$, Wang $\mathrm{Y}, \mathrm{Hu} \mathrm{K}$, et al. WGCNA reveals key gene modules regulated by the combined treatment of colon cancer with PHY906 and CPT11. Biosci Rep. 2020;40(9). doi:10.1042/BSR20200935.

13. Yang R, Du Y, Wang L, Chen Z, Liu X. Weighted gene co-expression network analysis identifies CCNA2 as a treatment target of prostate cancer through inhibiting cell cycle. $J$ Cancer. 2020;11 (5):1203-1211. doi:10.7150/jca.38173

14. Xue K, Cao J, Wang Y, et al. Identification of potential therapeutic gene markers in nasopharyngeal carcinoma based on bioinformatics analysis. Clin Transl Sci. 2020;13(2):265-274. doi:10.1111/cts. 12690

15. Kumar S, Kaushik A, Narasimhan B, et al. Molecular docking, synthesis and biological significance of pyrimidine analogues as prospective antimicrobial and antiproliferative agents. BMC Chem. 2019;13(1):85. doi:10.1186/s13065-019-0601-z

16. Langfelder P, Horvath S. WGCNA: an R package for weighted correlation network analysis. BMC Bioinform. 2008;9:559. doi:10.1186/1471-2105-9-559

17. Lopes CT, Franz M, Kazi F, et al. Cytoscape web: an interactive web-based network browser. Bioinformatics. 2010;26 (18):2347-2348. doi:10.1093/bioinformatics/btq430

18. Chin $\mathrm{CH}$, Chen $\mathrm{SH}, \mathrm{Wu} \mathrm{HH}$, et al. cytoHubba: identifying hub objects and sub-networks from complex interactome. Bmc Syst Biol. 2014;8(Suppl 4):S11. doi:10.1186/17520509-8-S4-S11

19. Li C, Xu J. Feature selection with the Fisher score followed by the Maximal Clique Centrality algorithm can accurately identify the hub genes of hepatocellular carcinoma. Sci Rep. 2019;9(1):17283. doi:10.1038/s41598-019-53471-0 
20. Zhang L, Ren B, Zhang J, et al. Anti-tumor effect of Scutellaria barbata D. Don extracts on ovarian cancer and its phytochemicals characterisation. J Ethnopharmacol. 2017;206:184-192. doi:10.1016/ j.jep.2017.05.032

21. Dai ZJ, Wang BF, Lu WF, et al. Total flavonoids of Scutellaria barbata inhibit invasion of hepatocarcinoma via MMP/TIMP in vitro. Molecules. 2013;18(1):934-950. doi:10.3390/ molecules18010934

22. Kan X, Zhang W, You R, et al. Scutellaria barbata D. Don extract inhibits the tumor growth through down-regulating of Treg cells and manipulating Th1/Th17 immune response in hepatoma H22-bearing mice. BMC Complement Altern Med. 2017;17(1):41. doi:10.1186/ s12906-016-1551-9

23. Gong B, Kao Y, Zhang C, Sun F, Zhao H. Systematic investigation of Scutellariae barbatae herba for treating hepatocellular carcinoma based on network pharmacology. Evid Based Complement Alternat Med. 2018;2018:4365739. doi:10.1155/2018/4365739

24. Yasen M, Mizushima H, Mogushi K, et al. Expression of Aurora $\mathrm{B}$ and alternative variant forms in hepatocellular carcinoma and adjacent tissue. Cancer Sci. 2009;100(3):472-480. doi:10.1111/ j.1349-7006.2008.01068.x

25. Cai C, Wang W, Tu Z. Aberrantly DNA methylated-differentially expressed genes and pathways in hepatocellular carcinoma. $J$ Cancer. 2019;10(2):355-366. doi:10.7150/jca.27832

26. Sang L, Wang XM, Xu DY, Zhao WJ. Bioinformatics analysis of aberrantly methylated-differentially expressed genes and pathways in hepatocellular carcinoma. World J Gastroenterol. 2018;24 (24):2605-2616. doi:10.3748/wjg.v24.i24.2605

27. Agarwal R, Narayan J, Bhattacharyya A, Saraswat M, Tomar AK. Gene expression profiling, pathway analysis and subtype classification reveal molecular heterogeneity in hepatocellular carcinoma and suggest subtype specific therapeutic targets. Cancer Genet. 2017;216-217:37-51. doi:10.1016/j.cancergen.2017.06.002

28. Bi N, Sun Y, Lei S, et al. Identification of 40S ribosomal protein S8 as a novel biomarker for alcohol associated hepatocellular carcinoma using weighted gene coexpression network analysis. Oncol Rep. 2020;44(2):611-627. doi:10.3892/or.2020.7634

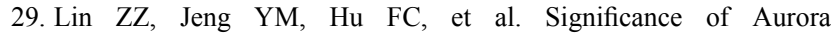
$\mathrm{B}$ overexpression in hepatocellular carcinoma. Aurora B overexpression in HCC. Bmc Cancer. 2010;10:461.

30. Carmena M, Ruchaud S, Earnshaw WC. Making the Auroras glow: regulation of Aurora A and B kinase function by interacting proteins. Curr Opin Cell Biol. 2009;21(6):796-805. doi:10.1016/j. ceb.2009.09.008

31. Lens SM, Wolthuis RM, Klompmaker R, et al. Survivin is required for a sustained spindle checkpoint arrest in response to lack of tension. EMBO J. 2003;22(12):2934-2947. doi:10.1093/emboj/ $\operatorname{cdg} 307$

32. Adams RR, Carmena M, Earnshaw WC. Chromosomal passengers and the (aurora) ABCs of mitosis. Trends Cell Biol. 2001;11 (2):49-54. doi:10.1016/S0962-8924(00)01880-8
33. Adams RR, Wheatley SP, Gouldsworthy AM, et al. INCENP binds the Aurora-related kinase AIRK2 and is required to target it to chromosomes, the central spindle and cleavage furrow. Curr Biol. 2000;10(17):1075-1078. doi:10.1016/S0960-9822(00)00673-4

34. Chen L, Chao SB, Wang ZB, et al. Checkpoint kinase 1 is essential for meiotic cell cycle regulation in mouse oocytes. Cell Cycle. 2012;11(10):1948-1955. doi:10.4161/cc.20279

35. Tun JO, Salvador-Reyes LA, Velarde MC, et al. Synergistic cytotoxicity of renieramycin $\mathrm{M}$ and doxorubicin in MCF-7 breast cancer cells. Mar Drugs. 2019;17(9):536. doi:10.3390/md17090536

36. Gong D, Feng PC, Ke XF, et al. Silencing long non-coding RNA LINC01224 inhibits hepatocellular carcinoma progression via microRNA-330-5p-induced inhibition of CHEK1. Mol Ther Nucleic Acids. 2020;19:482-497. doi:10.1016/j.omtn.2019.10.007

37. Bao J, Yu Y, Chen J, et al. MiR-126 negatively regulates PLK-4 to impact the development of hepatocellular carcinoma via ATR/ CHEK1 pathway. Cell Death Dis. 2018;9(10):1045. doi:10.1038/ s41419-018-1020-0

38. Fry AM, Schultz SJ, Bartek J, Nigg EA. Substrate specificity and cell cycle regulation of the Nek2 protein kinase, a potential human homolog of the mitotic regulator NIMA of Aspergillus nidulans. J Biol Chem. 1995;270(21):12899-12905. doi:10.1074/ jbc. 270.21.12899

39. Chang YY, Yen CJ, Chan SH, et al. NEK2 promotes hepatoma metastasis and serves as biomarker for high recurrence risk after hepatic resection. Ann Hepatol. 2018;17(5):843-856. doi:10.5604/ 01.3001 .0012 .3146

40. Zhang Y, Wang W, Wang Y, et al. NEK2 promotes hepatocellular carcinoma migration and invasion through modulation of the epithelial-mesenchymal transition. Oncol Rep. 2018;39 (3):1023-1033. doi:10.3892/or.2018.6224

41. Faragher AJ, Fry AM. Nek2A kinase stimulates centrosome disjunction and is required for formation of bipolar mitotic spindles. Mol Biol Cell. 2003;14(7):2876-2889. doi:10.1091/mbc.e03-02-0108

42. Lee J, Gollahon L. Nek2-targeted ASO or siRNA pretreatment enhances anticancer drug sensitivity in triple negative breast cancer cells. Int J Oncol. 2013;42(3):839-847. doi:10.3892/ijo.2013.1788

43. Hayward DG, Clarke RB, Faragher AJ, et al. The centrosomal kinase Nek2 displays elevated levels of protein expression in human breast cancer. Cancer Res. 2004;64(20):7370-7376. doi:10.1158/00085472.CAN-04-0960

44. Zeng X, Shaikh FY, Harrison MK, et al. The Ras oncogene signals centrosome amplification in mammary epithelial cells through cyclin D1/Cdk4 and Nek2. Oncogene. 2010;29(36):5103-5112. doi:10.1038/onc.2010.253

45. Wu SM, Lin SL, Lee KY, et al. Hepatoma cell functions modulated by NEK2 are associated with liver cancer progression. Int J Cancer. 2017;140(7):1581-1596. doi:10.1002/ijc.30559
International Journal of General Medicine

\section{Publish your work in this journal}

The International Journal of General Medicine is an international, peer-reviewed open-access journal that focuses on general and internal medicine, pathogenesis, epidemiology, diagnosis, monitoring and treatment protocols. The journal is characterized by the rapid reporting of reviews, original research and clinical studies across all disease areas. The manuscript management system is completely online and includes a very quick and fair peer-review system, which is all easy to use. Visit http://www.dovepress.com/ testimonials.php to read real quotes from published authors. 